\title{
Mechanobiology of Cutaneous Scarring
}

Rei Ogawa

\section{Contents}

\subsection{Background - 12}

2.2 Role of Mechanobiology in Cutaneous Scarring - 12

2.3 Cellular and Tissue Responses to Mechanical Forces - 12

2.4 Role of Mechanobiology in the Development of Pathological Scars - 13

2.5 A Pathological Scar Animal Model that Is Based on Mechanotransduction - 16

2.6 Mechanotherapy for Scar Prevention and Treatment - 16

2.6.1 Stabilization Materials - 16

2.6.2 Sutures - 16

2.6.3 Z-Plasty, Skin Grafting, and Local Flaps - 17

2.7 Conclusion - 17

References -17 


\subsection{Background}

The last phase of cutaneous wound healing produces the scar. Scars mainly consist of dermal-like collagens that are covered by the epidermis. The early stage of scarring is marked by inflammation, whose purpose is to close the wound gap. The source of the inflammation in the wound is the blood vessels, which exhibit vascular permeability after wounding. This allows the influx of inflammatory soluble factors and many types of immune cells from the circulation into the wound bed. Moreover, in the early stages of wound healing, resident cells, including collagen-secreting fibroblasts, accumulate in the damaged area. Finally, collagens, blood vessels, and nerve fibers are produced, resulting in an immature scar that is red, elevated, hard, and painful.

\subsection{Role of Mechanobiology in Cutaneous Scarring}

Under normal circumstances, the immature scar then undergoes the scar maturation process over several months. This process involves tissue remodeling, which associates with a natural decrease in the inflammation and the numbers of blood vessels, collagen fibers, and fibroblasts. However, sometimes the scar maturation process is not properly engaged because inflammation continues in the scar. Consequently, the immature scar stage is prolonged. This results in the pathological scars called hypertrophic scars and keloids. Many factors that prolong the inflammatory stage have been identified [1]. However, multiple lines of evidence acquired in recent years suggest that mechanical force can be an important cause of pathological scar development (some of these lines of evidence will be described in more detail below) [2]. This notion is also supported by the empirically acquired clinical understanding that noticeable scarring can be prevented by stabilizing surgical wounds with sutures and postsurgical dressings: the sutures help to approximate the wound edges with a small amount of intrinsic tension, while the dressings suppress the extrinsic mechanical forces on the wound/scar edges [3]. When the mechanical forces on and in the scar are imbalanced, a heavy scar can result.

\subsection{Cellular and Tissue Responses to Mechanical Forces}

The mechanical forces on scars/wounds include stretching tension, shear force, scratch, compression, hydrostatic pressure, and osmotic pressure. These forces are

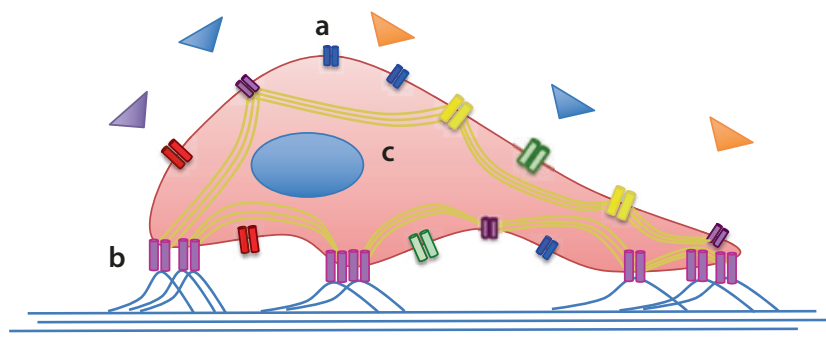

- Fig. 2.1 Schematic depiction of the mechanosensors that allow cells to sense extrinsic mechanical forces. (A) Mechanosensitive ATP hemichannels and $\mathrm{Ca}^{2+}$ ion channels. (B) Mechanosensitive molecules on the cell membrane, including cell adhesion molecules. (C) Cytoskeletal elements, including the actin filaments. When the extracellular matrix is distorted by mechanical forces such as skin tension (purple, blue, and orange triangles indicate different mechanical forces), mechanosensors on and in the resident cells sense the forces and initiate mechanosignaling pathways that lead to a large variety of molecular biological changes, including gene expression. The mechanosensors include mechanosensitive cell membrane molecules such as ATP hemichannels and $\mathrm{Ca}^{2+}$ ion channels (A, blue, red, and green cylinder pairs). Others are cell adhesion molecules such as integrin (B, purple cylinder pairs). Cytoskeletal elements such as the actin filaments $(\mathbf{C}$, the beige ropelike bundles within the cell) also play an important role in sensing and responding to extrinsic mechanical forces

perceived by mechanosensors on and in the cells that reside in the extracellular matrix as well as by nerve fiber mechanoreceptors at the tissue level $[4,5]$.

The cellular mechanosensors include mechanosensitive cell membrane molecules such as ATP hemichannels, $\mathrm{Ca}^{2+}$ ion channels, and cell adhesion molecules such as integrin (• Fig. 2.1). Cytoskeletal components such as actin filaments also sense mechanical force: when they do, actin polymerization and depolymerization occur [6]. Thus, when the extracellular matrix is distorted by mechanical forces such as skin tension, the cellular mechanosensors are triggered and initiate mechanosignaling pathways that lead to a variety of molecular biological changes [7], including gene expression, cell proliferation, angiogenesis, and epithelialization. The key mechanosignaling pathways [8] that are involved in scarring at the cellular level appear to be the integrin, MAPK/G protein, $\mathrm{TNF}-\alpha / \mathrm{NF}-\kappa \mathrm{B}, \mathrm{Wnt} / \beta$-catenin, interleukin, calcium ion, TGF $\beta / \mathrm{Smad}$, and FAK signaling pathways [9] (• Fig. 2.2).

At the tissue level, sensory nerve fibers, namely, mechanosensitive nociceptors, in the skin act as mechanoreceptors and thereby produce the somatic sensation of mechanical force [5]. Thus, when mechanical stimuli are received by mechanosensitive nociceptors somewhere on the body, electrical signals are transmitted to the dorsal root ganglia, which contain the neuronal cell bodies of the afferent spinal nerves ( Fig. 2.3). This causes the peripheral terminals of the primary afferent 


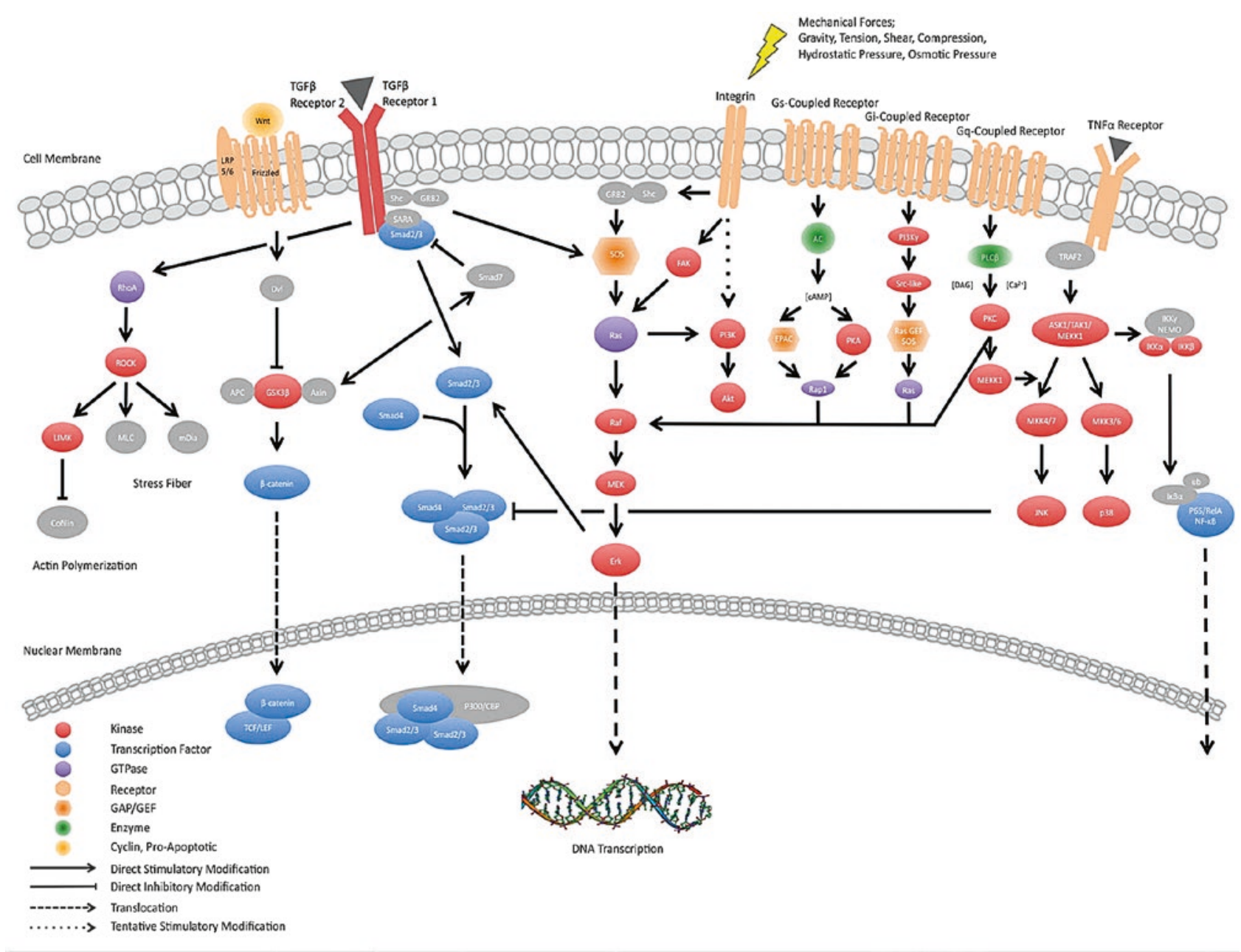

- Fig. 2.2 Mechanosignaling pathways in cells subjected to mechanical forces. When the mechanosensors in or on the cells are triggered, they activate various mechanosignaling pathways that

sensory neurons that innervate the skin to release neuropeptides, including substance $P$, calcitonin gene-based peptide (CGRP), neurokinin A, vasoactive intestinal peptide, and somatostatin. Since the peripheral terminals of the neuropeptide-releasing neurons are often in physical contact with cells in the wound/scar, the neuropeptides can directly target keratinocytes, fibroblasts, Langerhans, mast, dermal microvascular endothelium, and infiltrating immune cells. This can alter cell proliferation, cytokine production, antigen presentation, sensory neurotransmission, mast cell degradation, and vasodilation. The triggering of mechanonociceptors is particularly known to increase vascular permeability under both physiological and pathophysiological conditions. All of these pro-inflammatory responses produce what is termed neurogenic inflammation $[4,5]$. Notably, substance $\mathrm{P}$ and CGRP, which, respectively, act through the neurokinin 1 receptor and CGRP1 receptor, are synthesized during nerve growth factor regulation. It has been suggested that neurogenic inflammation/neu- then regulate cell proliferation, angiogenesis, and epithelialization. (This figure is from reference [8] with copyright permission from the publisher. (C) All rights reserved)

ropeptide activity plays a role in the development of burn and abnormal scars such as keloids and hypertrophic scars [10].

\subsection{Role of Mechanobiology in the Development of Pathological Scars}

Traditionally, hypertrophic scars and keloids are diagnosed as separate clinical and pathological entities, even though both are characterized by prolonged and aberrant extracellular matrix accumulation. The so-called typical keloids grow beyond the confines of their original wounds and exhibit accumulation of dermal thick eosinophilic collagen bundles with dermal nodules under the microscope. By contrast, hypertrophic scars generally grow within the boundaries of wounds and are characterized histologically by dermal nodules alone. 


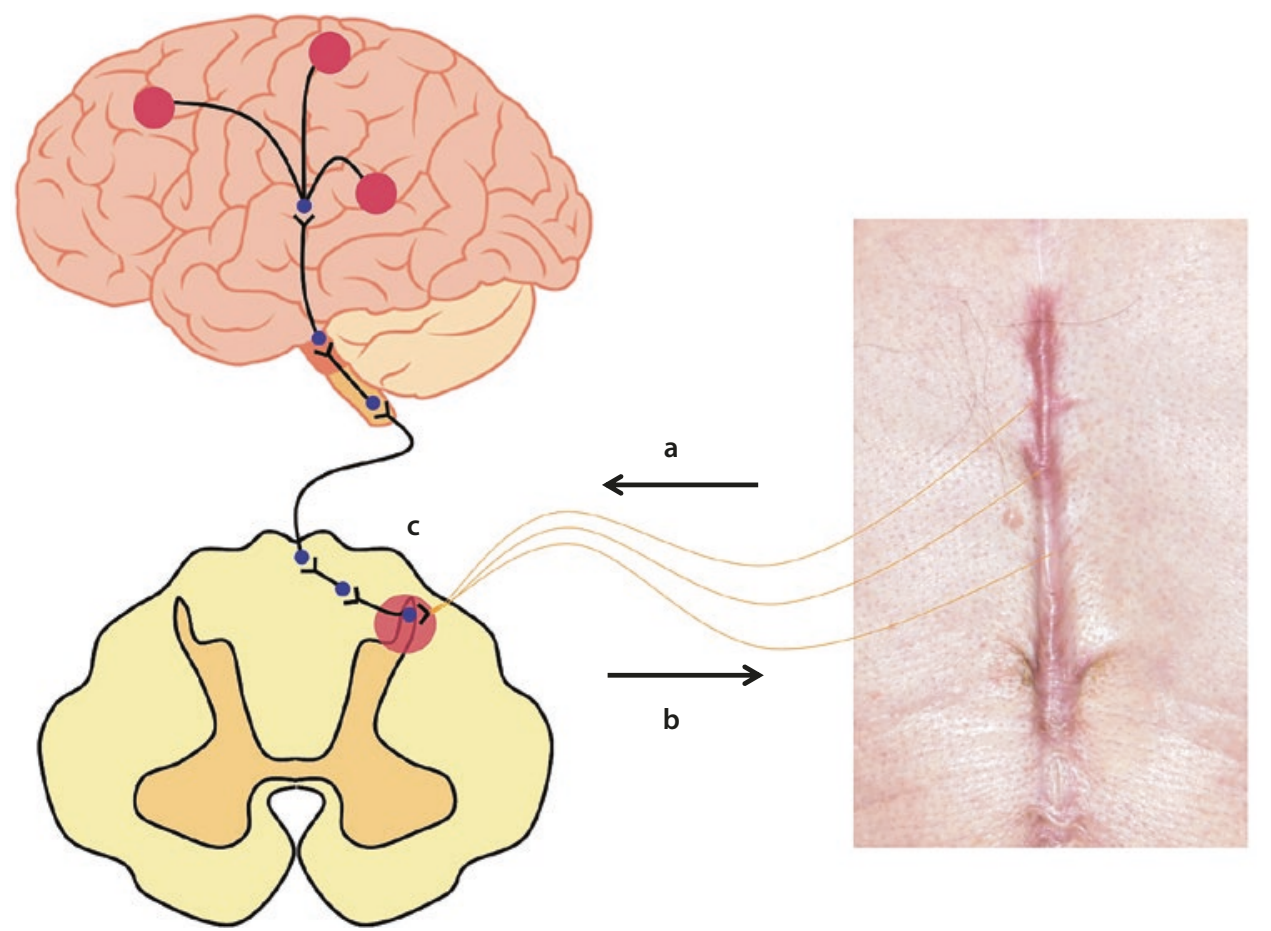

- Fig. 2.3 Mechanosensitive nociceptors shape somatic sensations and tissue responses to mechanical forces. Tissues such as skin respond to external mechanical forces such as tension via mechanosensitive nociceptors, whose neuronal cell bodies are located in the dorsal root ganglia in the spinal cord c. Thus, when a mechanical force is placed on a scar, the mechanosensitive nociceptors in and around the scar convert the mechanical stimuli into electrical signals that travel to the dorsal root ganglia in the spinal cord and then to

the brain a. This produces the somatic sensations to the mechanical force (e.g., pain and/or itch). Simultaneously, electrical signals return from the dorsal root ganglia to the skin mechanosensitive nociceptors $\mathbf{b}$. This causes them to release neuropeptides from their peripheral terminals. Since these terminals are often in physical contact with cells in the scar, including epidermal and dermal cells, the neuropeptides can induce neurogenic inflammation, thereby promoting pathological scar formation and progression

One of the reasons why hypertrophic scars and keloids are considered to be separate clinical and pathological entities is that keloids are relatively rare in the Caucasian population, which may have limited clinical experience with these scars. However, even senior clinicians sometimes have difficulty in differentiating between the two conditions, particularly with the so-called atypical cases that bear characteristics of both scar types. This is particularly true in Asian countries, where keloids are very common. We currently believe that hypertrophic scars and keloids represent successive stages or alternative forms of the same underlying fibroproliferative pathological lesion and that their progression or development into one or the other classical form may be determined by a variety of pro-inflammatory risk factors (- Fig. 2.4) $[11,12]$. One of these is mechanical forces. The importance of this factor in pathological scarring is supported by a number of observations, including the fact that hypertrophic scars can be generated in experimental animal models by mechanical forces [13]. Moreover, an analysis of Asian patients showed that keloids tend to occur at specific sites (the anterior chest, shoulder, scapular, and lower abdomen-suprapubic regions) that are

constantly or frequently subjected to mechanical forces, including skin stretching due to daily body movements [14]. Thus, the anterior chest skin is regularly stretched horizontally by the upper limb movements, the shoulder and scapula skin is constantly stretched by upper limb movements and body bending motions, and the lower abdomen and suprapubic skin regions are stretched hundreds of time a day by sitting and standing motions. Conversely, heavy scars rarely develop on the scalp, upper eyelid, and anterior lower leg, even in patients with extensive keloids or hypertrophic scars that cover much of the body. This pattern is likely to reflect the absence of tension on the skin in these regions. Thus, even in cases of deep wounding on the scalp and anterior lower leg, there is little tension on the wound because the skin on these regions is stabilized by the bones that lie directly below it. Moreover, there is little tension on the upper eyelid during the opening and closing of the eyes.

Another key piece of evidence that indicates the importance of mechanical forces in pathological scar formation and progression is the fact that keloids grow horizontally in the direction(s) of the predominant 

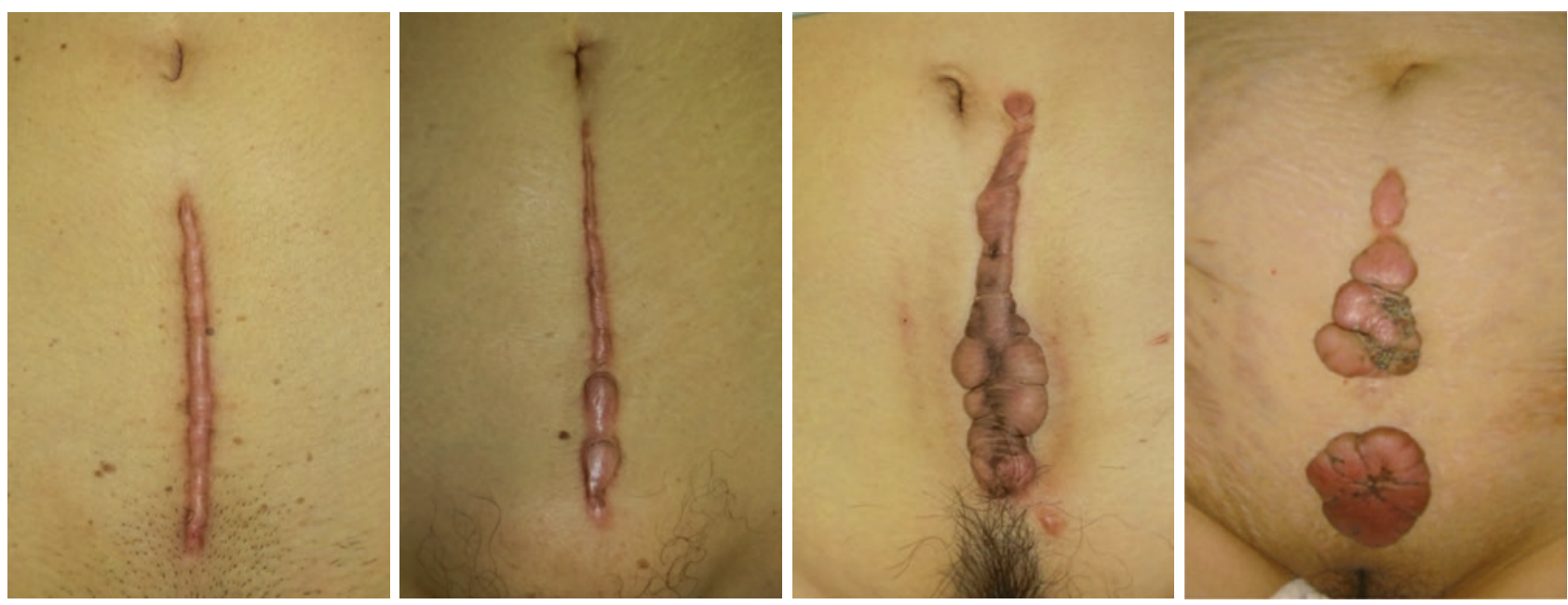

- Fig. 2.4 Difference between hypertrophic scars and keloids. The main difference between keloids and hypertrophic scars is that keloids have stronger and more prolonged inflammation. a depicts the strength and/or duration of inflammation

- Fig. 2.5 Typical shape of keloids on specific regions of the body. a The "butterfly" shape on the anterior chest. b The "crab's claw" shape on the anterior chest. c The "dumbbell" shape on the upper arm. These site-specific keloid shapes reflect the predominant directions of skin tension in these sites

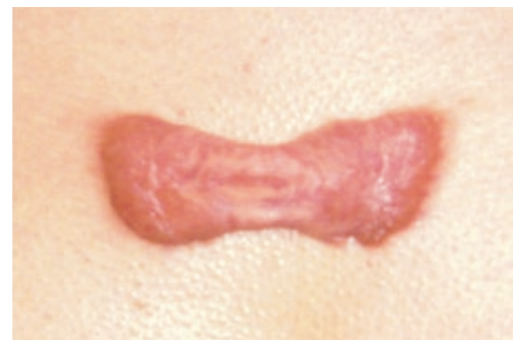

a

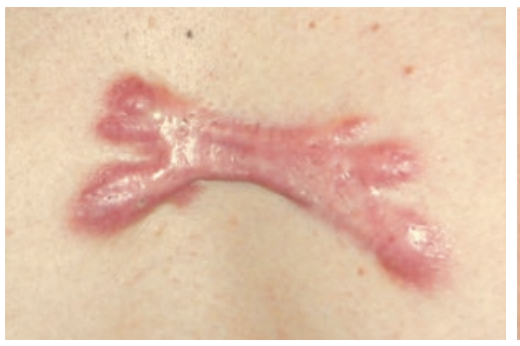

b

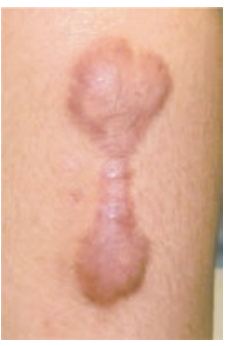

C forces on the wound/scar. This results in characteristic keloid shapes on specific locations. For example, keloids on the anterior chest grow in a "crab's claw" or "butterfly" shape, whereas upper arm keloids grow in a "dumbbell"-like shape along the long axis of the limb (• Fig. 2.5).

Keloids exhibit stronger and more prolonged inflammation than hypertrophic scars. This together with all of the mechanobiological observations described above suggests that keloid and hypertrophic scars largely differ because of the degree of skin tension on the wound/scar, which in turn determines the degree of inflammation (- Fig. 2.4). Thus, pronounced and highly repetitive skin tension on the wound may lead to greater inflammation and keloid formation, while less strong or different mechanical forces lead to a weaker or qualitatively different inflammation that leads to hypertrophic scar formation. This is supported by our previous finite element analysis of the mechanical force distribution around keloids [15], which showed that both the skin tension on the keloid and the inflammation within the keloid are particularly high at the leading keloid edges (- Fig. 2.6). Thus, the mechanical forces coming from the predominant direction(s) drive high inflammation at the leading keloid edges: this provokes local collagen production, which in turn causes the keloid to invade further in the direction of the skin tension.

It should be noted, however, it is highly likely that the inflammatory status in heavy scars is also shaped by many other risk factors, including local, genetic, and systemic factors such as hypertension (high blood pressure) [16]. 
- Fig. 2.6 Distribution of the mechanical forces around keloids. a A scapular keloid. b Finite element analysis of the mechanical force on the scapular keloid. The inflamed and elevated portions of the keloid a match with the areas of high tension in the keloid (red color in b). This suggests that high skin tension prolongs and amplifies the inflammation in the periphery of keloids. (The figure is from reference [15] with copyright permission from the publisher. (C) All rights reserved)
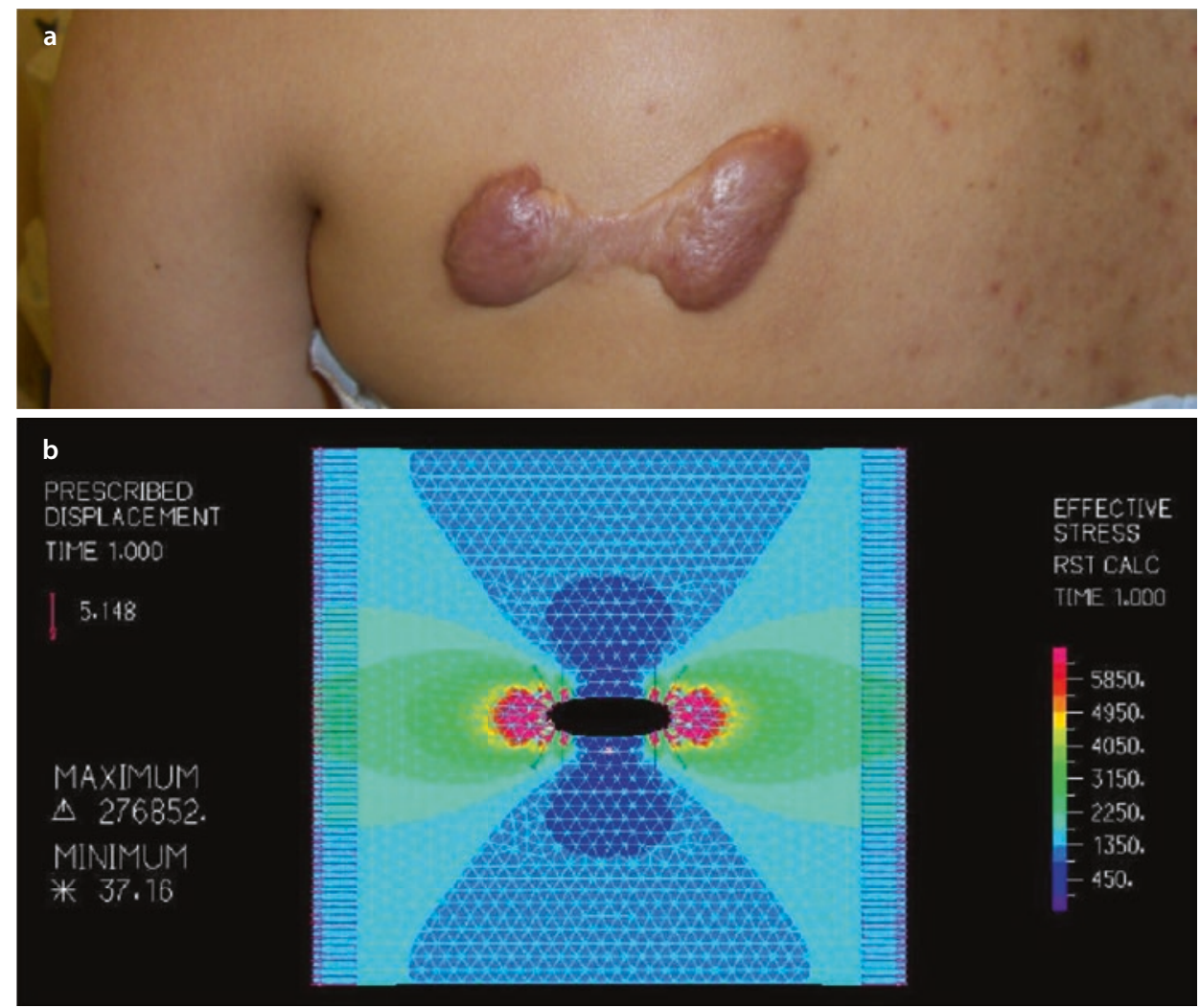

\subsection{A Pathological Scar Animal Model that Is Based on Mechanotransduction}

Many researchers have sought to develop animal models of heavy scars by using mice, rats, and rabbits. It should be noted that the scars of all of these models, especially the keloid models, seem to be driven more by an acute inflammatory response than by chronic inflammation: consequently, the resulting scars are largely immature. Nevertheless, an interesting model is the hypertrophic scar mouse model in which heavy scars are induced by placing a mechanical force on the edges of a cutaneous incision. Analysis of this model shows that when scars are subjected to tension, they exhibit less apoptosis and that inflammatory cells and mechanical forces promote fibrosis [13]. In addition, studies with this model show that during pathological scar development, cellular mechanosignaling pathways interact actively with the extracellular matrix and crosstalk extensively with the hypoxia, inflammation, and angiogenesis pathways.

\subsection{Mechanotherapy for Scar Prevention and Treatment}

\subsubsection{Stabilization Materials}

To limit skin stretching and external mechanical stimuli during wound healing/scarring, wounds and scars should be covered by fixable materials such as tape, bandages, garments, and silicone gel sheets. Several randomized controlled trials have shown that such wound stabilization reduces the incidence of hypertrophic scars or keloids. Our computer analysis of the mechanical forces around scars also showed that silicone gel sheeting reduces the tension at the scar edges [17].

\subsubsection{Sutures}

The fact that mechanical tension promotes keloid and hypertrophic scar development together with the fact that these scars arise from the dermis (especially the reticular dermis) suggests that closing surgical 
wounds with sutures that place little tension on the wound dermis may reduce the risk of pathological scar formation after surgery. Therefore, we use subcutaneous/fascial tensile reduction sutures that place the tension on the deep fascia and superficial fascia layer rather than the dermis (see $>$ Chap. 21). This minimizes the use of dermal sutures; in fact, dermal sutures can be avoided altogether if the wound edges approximate each other closely after the fascial sutures are placed.

\subsubsection{Z-Plasty, Skin Grafting, and Local Flaps}

In scar revision surgery, it is important to choose the surgical approach that most effectively reduces tension on the scar. An excellent choice is the Z-plasty, which disrupts the line of tension on linear scars (see - Chap. 21). Another choice is to use skin grafts. Fullthickness skin grafts (FTSG) are a better choice than split-thickness skin grafts (STSG) because the latter associate with a greater risk of secondary contracture. However, if adjacent normal skin is available, it is better to use local flaps rather than FTSG because they have a much lower risk of contracture and better esthetic outcomes. Once the decision to use a local flap has been made, one then has to decide between an island flap and a skin-pedicled flap. While this choice depends somewhat on the scar geometry and other patient-specific variables, skin-pedicled flaps are generally the better choice because they have greater extensibility than island flaps and are therefore good for releasing the tension of a scar (see $>$ Chap. 21). This greater extensibility probably reflects the fact that the entire perimeter of the island flap is surrounded by new scar whereas the skinpedicled flap maintains a connection with normal skin, which is much more elastic than scar tissue.

\subsection{Conclusion}

It is suggested that mechanical forces on the skin strongly influence the cellular behavior that leads to scarring. These observations led us to focus on the importance of reducing skin tension when keloids and hypertrophic scars are surgically removed to prevent their recurrence. Clinical studies revealed that subcutaneous/fascial tensile reduction sutures, which apply minimal tension on the dermis, are effective in reducing recurrence. Moreover, we have found that by using Z-plasty and skin flaps, which release tension on the wound, huge scars can be successfully treated.

\section{Take-Home Messages}

- Mechanical force can be an important cause of pathological scar development.

- The mechanical forces on scars/wounds include stretching tension, shear force, scratch, compression, hydrostatic pressure, and osmotic pressure.

- An evidence that indicates the importance of mechanical forces in pathological scar formation and progression is the fact that keloids grow horizontally in the direction(s) of the predominant forces on the wound/scar.

- There is the hypertrophic scar mouse model in which heavy scars are induced by placing a mechanical force on the edges of a cutaneous incision.

\section{References}

1. Huang C, Murphy GF, Akaishi S, Ogawa R. Keloids and hypertrophic scars: update and future directions. Plast Reconstr Surg Glob Open. 2013;1(4):e25.

2. Ogawa R. Mechanobiology of scarring. Wound Repair Regen. 2011;19(Suppl 1):s2-9.

3. Ogawa R, Akaishi S, Huang C, Dohi T, Aoki M, Omori Y, Koike S, Kobe K, Akimoto M, Hyakusoku H. Clinical applications of basic research that shows reducing skin tension could prevent and treat abnormal scarring: the importance of fascial/subcutaneous tensile reduction sutures and flap surgery for keloid and hypertrophic scar reconstruction. J Nippon Med Sch. 2011;78(2):68-76.

4. Ogawa R. Keloid and hypertrophic scarring may result from a mechanoreceptor or mechanosensitive nociceptor disorder. Med Hypotheses. 2008;71(4):493-500.

5. Akaishi S, Ogawa R, Hyakusoku H. Keloid and hypertrophic scar: neurogenic inflammation hypotheses. Med Hypotheses. 2008;71(1):32-8.

6. Hayakawa K, Tatsumi H, Sokabe M. Actin filaments function as a tension sensor by tension-dependent binding of cofilin to the filament. J Cell Biol. 2011;195(5):721-7.

7. Tatsumi H, Furuichi T, Nakano M, Toyota M, Hayakawa K, Sokabe M, Iida H. Mechanosensitive channels are activated by stress in the actin stress fibres, and could be involved in gravity sensing in plants. Plant Biol (Stuttg). 2014;16(Suppl 1):18-22.

8. Huang C, Akaishi S, Ogawa R. Mechanosignaling pathways in cutaneous scarring. Arch Dermatol Res. 2012;304(8):589-97.

9. Wong VW, Rustad KC, Akaishi S, Sorkin M, Glotzbach JP, Januszyk M, Nelson ER, Levi K, Paterno J, Vial IN, Kuang AA, Longaker MT, Gurtner GC. Focal adhesion kinase links mechanical force to skin fibrosis via inflammatory signaling. Nat Med. 2011;18(1):148-52. 
10. Scott JR, Muangman P, Gibran NS. Making sense of hypertrophic scar: a role for nerves. Wound Repair Regen. 2007;15(Suppl 1):S27-31.

11. Huang C, Akaishi S, Hyakusoku H, Ogawa R. Are keloid and hypertrophic scar different forms of the same disorder? A fibroproliferative skin disorder hypothesis based on keloid findings. Int Wound J. 2014;11(5):517-22.

12. Ogawa R, Akaishi S, Kuribayashi S, Miyashita T. Keloids and hypertrophic scars can now be cured completely: recent progress in our understanding of the pathogenesis of keloids and hypertrophic scars and the most promising current therapeutic strategy. J Nippon Med Sch. 2016;83(2):46-53.

13. Aarabi S, Bhatt KA, Shi Y, Paterno J, Chang EI, Loh SA, Holmes JW, Longaker MT, Yee H, Gurtner GC. Mechanical load initiates hypertrophic scar formation through decreased cellular apoptosis. FASEB J. 2007;21(12):3250-61.
14. Ogawa R, Okai K, Tokumura F, Mori K, Ohmori Y, Huang C, Hyakusoku H, Akaishi S. The relationship between skin stretching/contraction and pathologic scarring: the important role of mechanical forces in keloid generation. Wound Repair Regen. 2012;20(2):149-57.

15. Akaishi S, Akimoto M, Ogawa R, Hyakusoku H. The relationship between keloid growth pattern and stretching tension: visual analysis using the finite element method. Ann Plast Surg. 2008;60(4):445-51.

16. Arima J, Huang C, Rosner B, Akaishi S, Ogawa R. Hypertension: a systemic key to understanding local keloid severity. Wound Repair Regen. 2015;23(2):213-21.

17. Akaishi S, Akimoto M, Hyakusoku H, Ogawa R. The tensile reduction effects of silicone gel sheeting. Plast Reconstr Surg. 2010;126(2):109e-11e.

Open Access This chapter is licensed under the terms of the Creative Commons Attribution 4.0 International License (http://creativecommons. org/licenses/by/4.0/), which permits use, sharing, adaptation, distribution and reproduction in any medium or format, as long as you give appropriate credit to the original author(s) and the source, provide a link to the Creative Commons license and indicate if changes were made.

The images or other third party material in this chapter are included in the chapter's Creative Commons license, unless indicated otherwise in a credit line to the material. If material is not included in the chapter's Creative Commons license and your intended use is not permitted by statutory regulation or exceeds the permitted use, you will need to obtain permission directly from the copyright holder. 